\title{
Maintenance Intravenous Immunoglobulin Treatment for Multiple Sclerosis Coexisting with Ehlers-Danlos Syndrome and Muir-Torre Syndrome: A Case Study
}

\author{
Srishti Gupta $\cdot$ Mohnish Suri $\cdot$ Cris S. Constantinescu iD
}

Received: July 9, 2020 / Published online: August 11, 2020

(C) The Author(s) 2020

\begin{abstract}
The therapeutic options for disease modification in relapsing-remitting multiple sclerosis (RRMS) have expanded remarkably in the last 15 years. Although intravenous immunoglobulins (IVIg) have shown some therapeutic effects in multiple sclerosis, reducing global supplies, restriction of treatment to essential indications and availability of effective alternative treatments for MS currently exclude IVIg from being an accepted therapy for MS, other than for some exceptional considerations. We report the case of a female patient with RRMS who was diagnosed with Ehlers-Danlos syndrome (EDS) and Muir-Torre syndrome (MTS)
\end{abstract}

Digital Features To view digital features for this article go to https://doi.org/10.6084/m9.figshare.12759788.

S. Gupta $(\varangle)$. C. S. Constantinescu $(\bowtie)$

Department of Neurology, Queen's Medical Centre Campus, Nottingham University Hospitals NHS

Trust, Derby Road, Nottingham, UK

e-mail: srishti.gupta@nuh.nhs.uk

C. S. Constantinescu

e-mail: cris.constantinescu@nottingham.ac.uk

M. Suri

Clinical Genetics Service, City Hospital Campus, Nottingham University Hospitals NHS Trust,

Nottingham, UK

C. S. Constantinescu

Division of Clinical Neuroscience, Section of Clinical Neurology, University of Nottingham, Nottingham, UK soon after the diagnosis of active RRMS was made. The coexisting conditions precluded the use of available disease-modifying treatments. She benefited from monthly and then bi-monthly IVIg, with a single mild relapse over 10 years. Discontinuation of IVIg due to reduced availability with a brief aborted course of subcutaneous PEGylated interferon-beta was followed by significant relapses. Five months after the first ocrelizumab infusion, she developed caecal cancer requiring colectomy. Reinstitution of IVIg is contemplated.

Keywords: Ehlers-Danlos syndrome; IVIg; Muir-Torre syndrome; Multiple sclerosis

\section{Key Summary Points}

A complex case of RRMS with EDS and MTS-probable association/trigger/ unmasking

IVIg has been found beneficial as an immunomodulatory and potential cancer immunoprophylaxis agent

Unmet need of treatment options in this rare case-IVIg kept MS and cancer in remission

Despite the global shortage of IVIg, it should be considered for clinically indicated cases 


\section{INTRODUCTION}

The landscape for disease-modifying treatments for multiple sclerosis (MS), in particular for the relapsing-remitting forms, has changed dramatically over the last two decades. All current treatments target the immune system by reducing pro-inflammatory responses, blocking lymphocyte migration, providing non-specific immunosuppression or depleting selected immune cell populations.

Despite the evidence of a potential therapeutic benefit, intravenous immunoglobulin (IVIg) is currently only very rarely used in MS, where it is reserved for special situations, for example in the post-partum period to prevent post-partum relapses [1]. However, as resumption of disease-modifying treatments in the immediate post-partum period is increasingly accepted, IVIg use has declined even for this indication.

Secondary antibody deficiencies are increasingly diagnosed during the course of B cell-depleting therapies for central nervous inflammatory conditions, but these are typically seen with neuromyelitis optica spectrum disorders under rituximab treatment. Some of the affected patients benefit from IVIg given as prophylaxis against recurrent infections [2].

A major consideration in restricting the use of IVIg to conditions where alternatives are lacking is the declining supply of immunoglobulin worldwide, related to a reducing number of donors. This further excludes MS as an indication for IVIg other than for exceptional cases.

We report a unique case of a patient with relapsing-remitting multiple sclerosis (RRMS) whose constellation of co-morbidities (which included predisposition to cancer) precluded the use of the other available disease-modifying treatments. She benefited from monthly and then bi-monthly IVIg, with a single MS relapse and no recurrence of cancer in $>10$ years.

\section{CASE REPORT}

A 67-year old woman had optic neuritis at age 46 and was diagnosed with RRMS 1 year later.
She had three clinical relapses requiring corticosteroid treatment in the first 18 months of the disease. A short course of subcutaneous interferon beta 1a (Rebif $\left.^{\circledR}\right)$ was discontinued because of side effects (mood changes and severe bruising at the injection sites).

Three years after the first symptoms, she was recruited into a randomized placebo-controlled clinical trial of anti-IL-12 antibody for RRMS [3]. As part of the screening for the trial, a past personal and strong family history of hypermobility, increased skin elasticity and frequent joint dislocations was elicited, leading to the diagnosis of hypermobile Ehlers-Danlos syndrome (EDS), possibly with additional features (e.g., vascular fragility). Further family history was notable for endometrial adenocarcinoma in her mother and sister.

During the trial, she remained stable neurologically. Towards the end of the trial she developed a skin lesion on the left shoulder, which was biopsied and diagnosed as sebaceous adenoma.

This diagnosis taken together with her family history of endometrial cancer raised concerns about a diagnosis of Muir-Torre syndrome (MTS), which is a variant/subtype of Lynch syndrome [4]. Genetic testing showed that she was heterozygous for a constitutional pathogenic nonsense variant (p.(Lys537Ter) in the MSH2 gene. Screening for Lynch syndrome-related visceral malignancies was commenced. Soon after the end of the trial, where she had been allocated to the treatment arm (anti-IL-12 antibody), she was assessed for and confirmed as having endometrial carcinoma and hospitalized for hysterectomy and bilateral salpingo-oophorectomy. She suffered massive bleeding with hypovolemic shock during surgery. Excessive vascular fragility attributed to her EDS was noted during the surgery. After two severe MS relapses within 6 months, therapeutic choices were considered in multidisciplinary discussions. Beta-interferon and glatiramer acetate were avoided because of severe bruising with frequent injections. Immunosuppressants and natalizumab were ruled out in view of increased risk of MTS-associated malignancies during immunosuppression [5]. 
IVIg was considered the safest option, and the treatment was approved by the hospital's IVIg panel committee.

Due to vein fragility, a porta-cath was placed, and she was started on IVIg, $0.4 \mathrm{~g} / \mathrm{kg}$, one infusion every month for the first 2 years and then every 2 months subsequently. She had no further relapses for 3.5 years while being on IVIg except a short episode of oscillopsia lasting about 3 weeks, which resolved spontaneously. The MRI scan of the brain at that time did not show any new or acute lesions. She continued on IVIg with no relapses, no side effects and minimal disease progression (0.5 EDSS step) for a further 7 years. The frequency of UTI dropped to almost none during the IVIg treatment. She underwent routine periodic screening for malignancies throughout this period and did not develop new malignancy indicating the potential role of IVIg as an anti-cancer therapy $[6,7]$.

After 10 years, because of the global shortage of IVIg, the treatment was discontinued. After some deliberations, the patient agreed to start the fortnightly preparation of pegylated interferon beta 1 -a $\left(\right.$ Plegridy ${ }^{\circledR}$ ) in view of the low frequency of administration. After persevering for 6 months despite bruising at the injection site and low mood, she had increased suicidal ideation and hence stopped the interferon treatment. Two months later, she had a severe relapse with paraplegia requiring hospitalization and intravenous steroids. After her recovery, ocrelizumab, a B cell-depleting agent, was initiated. Five months after the first infusion, she was diagnosed with caecal carcinoma during a routine surveillance colonoscopy.

She underwent colectomy (with extensive precautions to prevent bleeding) with a stoma placement. In the post-operative period, she experienced a further significant relapse presenting with severe weakness and cerebellar dysfunction. She is currently rehabilitating, though not completely returned to her preoperative baseline. A reinstitution of IVIg has been requested and awaits approval.

\section{DISCUSSION}

We report a unique case of a patient with active RRMS who experienced significant relapses off treatment that were all but abolished during treatment with IVIg. In this case we had no choice but to give IVIg because of her coexisting conditions barring her from regular injectable drugs and immunosuppressive treatment.

Due to the increasing number and efficacy of therapeutic agents for MS, in addition to the global shortage, IVIg is no longer advocated as a treatment for MS. However, aside from these considerations, the body of evidence for IVIg is substantial. A meta-analysis by Sorensen et al. [8] combined four studies to assess the efficacy of IVIg in RRMS in a randomized, placebo-controlled manner. The study showed a significant beneficial effect on the annual relapse rate (effect size $-0.5 ; P=0.00003$ ) on the proportion of relapse-free patients $(0.29$ difference; $\left.P=2.1 \times 10^{-8}\right)$, change in the Expanded Disability Status Scale (EDSS) (effect size: 0.25; $P=0.04$ ) and a trend towards a reduction in the proportion of patients who deteriorated $(P=0.03)$. Each single study in the meta-analysis had its weaknesses, but all studies were positive regarding their primary end point, and the results yield concordant evidence for reduction of the relapse rate and progression [8].

Another meta-analysis by Olayeemanesh et al. [9] combined six studies using a parallelgroup design. The pooled results revealed significant differences in the number of relapses experienced on an annual basis in favour of IVIg $(\mathrm{SMD}=-0.218 ; 95 \% \mathrm{CI}-0.412$ to -0.024 ; $P=0.028)$ [9].

Ehlers-Danlos syndrome is not commonly associated with neurological manifestations. The most common are vascular features, such as aneurysm, subarachnoid haemorrhage and spontaneous arterial dissection. Various congenital malformations of the vessels and the brain parenchyma have been reported along with symptomatic and idiopathic epilepsy 
$[10,11]$. Our case series of four patients with MS and EDS showed joint laxity as the most prominent feature [12]. There have been case reports of EDS and leukodystrophy [13]. Extracellular matrix (ECM) proteins play an important role in CNS inflammation and myelination, endothelial lining, binding of soluble cytokines and adhesion of inflammatory cells, cellular migration and organization [14]. There is a suspicion that abnormalities in ECM protein can increase the susceptibility to autoimmune conditions [15]. Health care professionals need to be aware of several management issues in patients with MS and EDS such as increased risk of joint dislocation or arterial dissection during active physiotherapy [12].

Also, in this case due to EDS the skin can be very fragile and easily bruised, which would make any vascular access difficult. The patient was deemed unsuitable for subcutaneous injectable disease-modifying treatment because of skin fragility. Notably, there is a case series of two patients who had hypermobile EDS and MS/NMOSD-like CNS manifestations that were responsive to corticosteroids and plasmapheresis treatment. The authors found paraplegia more reversible in this category of patients compared to MS/NMOSD following immunotherapy [16].

Muir-Torre syndrome (MTS), an autosomal dominant condition caused by mutations in the mismatch repair genes, often $\mathrm{MSH} 2$, is part of the spectrum of Lynch syndrome (formerly called hereditary nonpolyposis colorectal cancer) that in addition presents sebaceous tumors including sebaceous adenoma. The onset of these tumors has been shown to be triggered or unmasked by immunocompromised states, either through pharmacological immunosuppression or through acquired immunodeficiency $[5,17,18]$. Once such a tumor is diagnosed, screening for visceral malignancies (colorectal and genito-urinary) and suspicion of MTS is essential. Avoidance of immunosuppressive therapies is also important.

An argument has been made for implementing immuno-interception using immune checkpoint inhibitors in MTS, following a report of a successful immuno-interception in a patient with 136 cutaneous and visceral tumours [19]. However, immune checkpoint inhibitors have been associated with a number of autoimmune neurological syndromes and can cause severe, even fatal, exacerbations of MS [20].

In our patient, it is tempting to speculate that IVIg, in addition to its sustained and thorough control of MS disease activity, may have also provided effective immuno-interception. The anti-tumor and anti-metastatic effect of IVIg has been recognized, and the occurrence of colon cancer after discontinuation of a 10-year-long course of IVIg may represent cessation of immuno-interception, more likely than an immunosuppressive effect of ocrelizumab, given that the patient only had one course of this drug.

In conclusion, despite the restrictions in the use of IVIg due to its globally reduced availability, this unique case illustrates a multitude of beneficial effects that warrant its continued use as a rare clinically indicated exception.

\section{ACKNOWLEDGEMENTS}

Funding. No funding or sponsorship was received for this study or publication of this article.

Authorship. All named authors meet the International Committee of Medical Journal Editors (ICMJE) criteria for authorship for this article, take responsibility for the integrity of the work as a whole, and have given their approval for this version to be published.

Disclosures. Srishti Gupta and Mohnish Suri have nothing to disclose. Cris S Constantinescu is an editorial board member for this journal.

Compliance with Ethics Guidelines. A written consent was obtained from the patient for the case to be published anonymously.

Data Availability. The datasets generated during and/or analyzed during the current study are available from the corresponding author on reasonable request. 
Open Access. This article is licensed under a Creative Commons Attribution-NonCommercial 4.0 International License, which permits any non-commercial use, sharing, adaptation, distribution and reproduction in any medium or format, as long as you give appropriate credit to the original author(s) and the source, provide a link to the Creative Commons licence, and indicate if changes were made. The images or other third party material in this article are included in the article's Creative Commons licence, unless indicated otherwise in a credit line to the material. If material is not included in the article's Creative Commons licence and your intended use is not permitted by statutory regulation or exceeds the permitted use, you will need to obtain permission directly from the copyright holder. To view a copy of this licence, visit http:// creativecommons.org/licenses/by-nc/4.0/.

\section{REFERENCES}

1. Haas J. High dose IVIG in the post partum period for prevention of exacerbations in MS. Mult Scler. 2000;6(Suppl 2):S18-S20 (discussion S33).

2. Tallantyre EC, Whittam DH, Jolles S, et al. Secondary antibody deficiency: a complication of antiCD20 therapy for neuroinflammation. J Neurol. 2018;265(5):1115-22.

3. Vollmer TL, Wynn DR, Alam MS, Valdes J. A phase 2, 24-week, randomized, placebo-controlled, double-blind study examining the efficacy and safety of an anti-interleukin-12 and -23 monoclonal antibody in patients with relapsing-remitting or secondary progressive multiple sclerosis. Mult Scler. 2011;17(2):181-91.

4. Shalin SC, Lyle S, Calonje E, et al. Sebaceous neoplasia and the Muir-Torre syndrome: important connections with clinical implications. Histopathology. 2010;56(1):133-47.

5. Landis MN, Davis CL, Bellus GA, Wolverton SE. Immunosuppression and sebaceous tumors: a confirmed diagnosis of Muir-Torre syndrome unmasked by immunosuppressive therapy. J Am Acad Dermatol. 2011;65:1054-8.

6. Tal S, Yehuda S. Uncovering the hidden potential of intravenous immunoglobulin as an anticancer therapy. Clin Rev Allergy Immunol. 2005;29: 307-10.

7. Xu Q, Zhang Z, Chen Z, et al. Nonspecific immunoglobulin $G$ is effective in preventing and treating cancer in mice. Cancer Manag Res. 2019;11:2073-85.

8. Sorensen PS, Fazekas F, Lee M. Intravenous immunoglobulin $G$ for the treatment of relapsing-remitting multiple sclerosis: a meta-analysis. Eur J Neurol. 2002;9:557-63.

9. Olyaeemanesh A, Rahmani M, Goudarzi R, et al. Safety and effectiveness assessment of intravenous immunoglobulin in the treatment of relapsingremitting multiple sclerosis: a meta-analysis. Med J Islam Repub Iran. 2016;30:336.

10. Jacome DE. Epilepsy in Ehlers-Danlos syndrome. Epilepsia. 1999;40:467-73.

11. Echaniz-Laguna A, de Saint-Martin A, Lafontaine $\mathrm{AL}$, et al. Bilateral focal polymicrogyria in EhlersDanlos syndrome. Arch Neurol. 2000;57:123-7.

12. Vilisaar J, Harikrishnan S, Suri M, Constantinescu CS. Ehlers-Danlos syndrome and multiple sclerosis: a possible association. Mult Scler. 2007;14: $1-4$.

13. Spranger S, Spranger M, Kirchhof K. Ehlers-Danlos syndrome type VIII and leukodystrophy. Am J Med Genet. 1996;66:239-40.

14. Sobel RA. The extracellular matrix in multiple sclerosis: an update. Braz J Med Biol Res. 2001;34: 603-9.

15. Gustafson A, Griswold B, Burchett ME, et al. Do abnormalities of extracellular matrix elements lead to autoimmune disorders? Unexpectedly high incidence of rheumatologic disorders in persons with Ehlers Danlos syndrome. In: Annual Meeting of the American Society of Human Genetics. New Orleans, Louisiana, 2006.

16. Araki $\mathrm{M}$, Lin $\mathrm{Y}$, Ono $\mathrm{H}$, et al. Application of immunotherapy for neurological manifestations in hypermobile Ehlers-Danlos syndrome. Ther Adv Neurol Disord. 2018;11:1-5.

17. Frantz S, Greiner A, Schoen C. A sebaceous tumor in a patient with acquired immunodeficiency syndrome. Eur J Med Res. 2002;7:135-7.

18. Stone MS, Duncan WC, McGavran MH. Torre's syndrome: exacerbation of cutaneous manifestations with immunosuppression. J Am Acad Dermatol. 1986;15:1001. 
19. Mancuso JG, Foulkes WD, Pollak MN. Cancer immunoprevention: a case report raising the possibility of "immuno-interception". Cancer Prev Res. 2020;13(4):351-6.
20. Garcia CR, Jayswal R, Adams V, Anthony LB, Villano JL. Multiple sclerosis outcomes after cancer immunotherapy. Clin Transl Oncol. 2019;21(10): 1336-422. 